\title{
Role of exercise in the treatment of alcohol use disorders (Review)
}

\author{
EIRINI MANTHOU ${ }^{1,2}$, KALLIOPI GEORGAKOULI $^{1,2}$, IOANNIS G. FATOUROS ${ }^{2}$, \\ CHRISTINA GIANOULAKIS $^{3}$, YANNIS THEODORAKIS ${ }^{1}$ and ATHANASIOS Z. JAMURTAS ${ }^{1,2}$ \\ ${ }^{1}$ Department of Physical Education and Sport Science, University of Thessaly; ${ }^{2}$ Department of Kinesiology, \\ Institute for Research and Technology Thessaly, 42100 Trikala, Greece; ${ }^{3}$ McGill University and \\ Douglas Mental Health University Institute, Montreal, Quebec H4H 1R3, Canada
}

Received December 9, 2015; Accepted February 9, 2016

DOI: 10.3892/br.2016.626

\begin{abstract}
Excessive alcohol use can cause harmful effects on the human body, which are associated with serious health problems, and it can also lead to the development of alcohol use disorders (AUDs). There is certain evidence that physical exercise positively affects excessive alcohol use and the associated problems by leading to reduced alcohol intake. A literature search was conducted using the databases PubMed, Medline and Web of Science. The search terms used as keywords were: Addiction, abuse, alcohol use disorders, exercise training, $\beta$-endorphin, opioids, brain, ethanol and alcohol. The current study presents the studies that reported on the use of exercise in the treatment of AUDs between 1970 and 2015. The potential psychological and physiological mechanisms that contribute to the action of exercise were also reviewed, highlighting the role of $\beta$-endorphin and the hypothalamic-pituitary-adrenal axis in AUDs and the possible association among physical activity, the endogenous opioid system and the desire for alcohol. Only 11 studies were identified that refer to the effect of exercise on alcohol consumption and/or the associated outcomes. Six of those studies concluded that exercise may have a positive impact towards alcohol consumption, abstinence rates or the urge to drink. One of those studies also indicated that a bout of exercise affects the endogenous opioids, which may be associated with the urge to drink. Another 3 studies indicated that responses to acute exercise in individuals with AUDs are different compared to those in healthy ones. Generally, despite limited research data and often contradictory results, there is certain early promising evidence for the role of exercise as an adjunctive tool in the treatment of AUDs. Physiological and biochemical parameters that would confirm that exercise is
\end{abstract}

Correspondence to: Dr Athanasios Z. Jamurtas, Department of Physical Education and Sport Science, University of Thessaly, 42100 Trikala, Greece

E-mail: ajamurt@pe.uth.gr

Key words: alcoholism, exercise training, $\beta$-endorphin, opioids, brain, ethanol safe for individuals with AUDs should be examined in future studies.

\section{Contents}

1. Introduction

2. Literature search

3. Results and Discussion

4. Conclusions

\section{Introduction}

The European Union has the highest percentages of alcohol drinkers worldwide, which hugely increases the health and socioeconomic burden of the member states (1). Excessive alcohol use is the third leading risk factor for disease following tobacco and blood pressure (2). The most important include peripheral neuropathy, brain damage, alcoholic liver disease, and nutritional and metabolic disorders. Furthermore, excessive alcohol use increases the likelihood of crime, accidents and violence, impulsivity and antisocial behavior (3). Due to the physiological, psychological and social consequences of alcohol use disorders (AUDs), there are a number of proposed treatment models for this issue outlined in the following.

According to the Diagnostic and Statistical Manual of Mental Disorders, Fifth Edition (DSM-V) (4), AUDs combines the terms of alcohol abuse and alcohol dependence in the revised chapter of 'Substance-Related and Addictive Disorders'. In brief, a substance use disorder is defined as a maladaptive pattern of substance use leading to clinically significant impairment or distress, as manifested by 2 (or more) of a list with 11 symptoms, occurring within a 12-month period. Alcohol abuse and alcohol dependence are combined into a single disorder measured on a continuum from mild to severe. Whereas a diagnosis of substance abuse previously required only 1 symptom, a substance use disorder in DSM-V requires $\geq 2$ symptoms from the list of 11 ; mild substance use disorder requires 2-3 symptoms and severe substance use disorder requires $\geq 4$.

In general, there are different approaches in the treatment of alcoholism. According to the Institute of Medicine (5), treatment for alcoholism takes place in three stages: The first stage 
involves detoxification, close monitoring and prompt treatment of the individual. The second stage involves restoring functioning, evaluation of the patient, provision of primary care and multidimensional monitoring. The third stage consists of maintaining the primary patient care, monitoring for reoccurrence and home care by specialists, according to the therapeutic model applied (5).

The detoxification procedure involves psychological and/or pharmacological support and an extensive follow-up period (6,7). Psychological management of alcoholism may include self-help groups, group therapy, psychotherapy and cognitive-behavioural therapy. These techniques deal with the underlying psychological issues that are associated with alcohol addiction, as well as providing relapse prevention skills. Another therapeutic approach to alcoholism widely used in the last 10 years is motivational interviewing (8). It is an approach that helps to increase the intrinsic motivation and commitment to treatment of the patient, enhancing the will to change. This technique applies to patients with extremely serious problems and those with less severe alcohol problems. The decision to change is stemming from the person with the help of intrinsic motivation, which is emphasized in the theory of self-determination (9). Aided by self-efficacy development through the procedure, the psychologist drives the interview in order for the patient to understand the difference between the ideal and the actual behavior without pushing for change or contradicting the person. The technique has been applied together with other approaches in the Matching Alcoholism Treatments to Client Heterogeneity Project $(10,11)$, one of the largest research programs to combat alcoholism within a sample of 1,726 patients. It is true, however, that addiction therapy is rarely linear and multiple relapses are inevitable.

Pharmacological treatment of alcoholism mostly involves altering the reinforcing effects of alcohol use (12). Medication development has focused on several neurotransmitter systems that interact with the cortico-mesolimbic dopamine pathway. A number of available or promising compounds appear to act by modulating the function of opioids, glutamate and serotonin. Naltrexone is a competitive antagonist for opioid receptors, which successfully blocks the effects of endorphins and opiates thereby preventing heavy drinking and decreases the urge to drink. Another treatment medication, odansetron, is a 5-hydroxytryptamine receptor antagonist also known as serotonin blocker and is effectively used mainly in patients with early-onset alcoholism and patients with a specific genetic variant of the serotonin transporter (5-HTT) gene. Acamprosate is a glutamate antagonist mainly used in the post-withdrawal phase. Other medicines, such as disulfiram and calcium carbimide, prevent the elimination of acetaldehyde and produce an unpleasant physical reaction $(6,7)$.

Exercise has been suggested as an alternative approach in the prevention or treatment of AUDs (13-15). Physical activity is unique in the sense that it is available to people who may not have access to other forms of treatment, such as psychological intervention or medication. It can also act as an alternative healthy activity versus addiction (16) and enhance mood and psychological wellbeing (17). However, treatment with the implementation of physical activity, as opposed to medication, requires the active participation of the individual and not the passive acceptance of treatment. It is internally driven and requires physical effort and commitment from the individual. For this reason, when the person is free to choose the type of exercise that is bound to follow, as well as its intensity, the treatment is more effective (18). Although it has been reported that exercise produces a feeling of wellbeing, the appropriate type or intensity of exercise that is required to improve mood remains to be elucidated and appears to depend on numerous factors (19). Additionally, there is limited research on the type and mode of exercise required to avert individuals with AUDs from alcohol consumption and how effective this type of treatment could be. The present study aimed to address the issue of AUDs by reviewing the research evidence on the therapeutic use of exercise in the treatment of these disorders and unveil possible underlying mechanisms.

\section{Literature search}

The PubMed, Medline and Web of Science databases were searched for studies in English published between 1970 and 2015, which had investigated any form of exercise interventions for the treatment of AUDs. Search terms included 'health', 'exercise', 'physical activity', 'substance use disorder,' 'dependence', 'abuse' and 'alcohol'. The references of all the retrieved studies were searched for additional references. Studies that exclusively referred to types of interventions other than exercise or did not report alcohol- or health-related results were excluded.

\section{Results and Discussion}

The literature search revealed 11 studies that fulfilled the inclusion criteria. Exercise mode, drinking patterns of the participants and research outcomes significantly varied among the studies. Only 4 of these studies used an acute intervention model, while the rest of them are medium- and long-term exercise intervention studies with a duration ranging from 4 to 15 weeks. Nine studies used alcoholic patients that had completed a detoxification program, whereas only 2 studies used heavy drinkers that were not diagnosed for any AUD. Furthermore, there has been limited research on exercise as an adjunctive strategy in AUDs treatment programs. To the best of our knowledge, only 8 studies have investigated the effect of exercise on alcohol consumption-related outcomes, which namely are direct alcohol consumption, abstinence rates and urge to drink. Research on the effects of exercise on biochemical indices is also scarce; only 4 studies have reported biochemical outcomes associated with exercise-induced hypothalamic-pituitary-adrenal (HPA) axis activation (Table I).

Acute exercise. Acute effects of exercise on alcohol urge and mood disturbance were examined in a counterbalanced cross-over design study (20). Twenty alcohol-dependent individuals, who had already completed a 3-month detoxification program, participated in the study. The experimental group underwent $10 \mathrm{~min}$ of moderate intensity exercise (40-60\% of the heart rate reserve), while the control group underwent 10 min of light intensity exercise (5-20\% of the heart rate reserve) in a cycle ergometer. The results showed that participants in the experimental group had a significantly reduced alcohol urge (by 19.7\%) compared to the 'control' group 


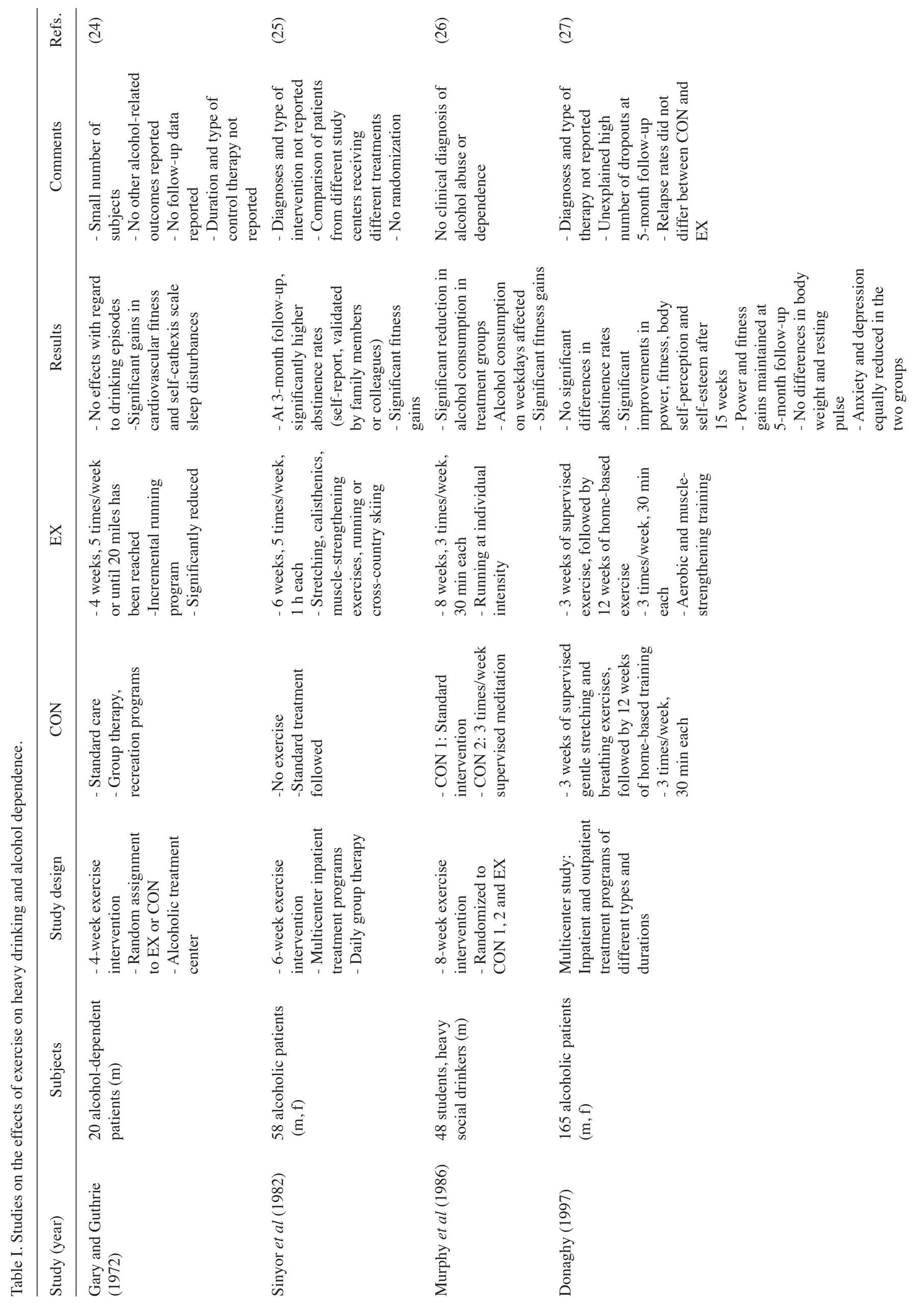




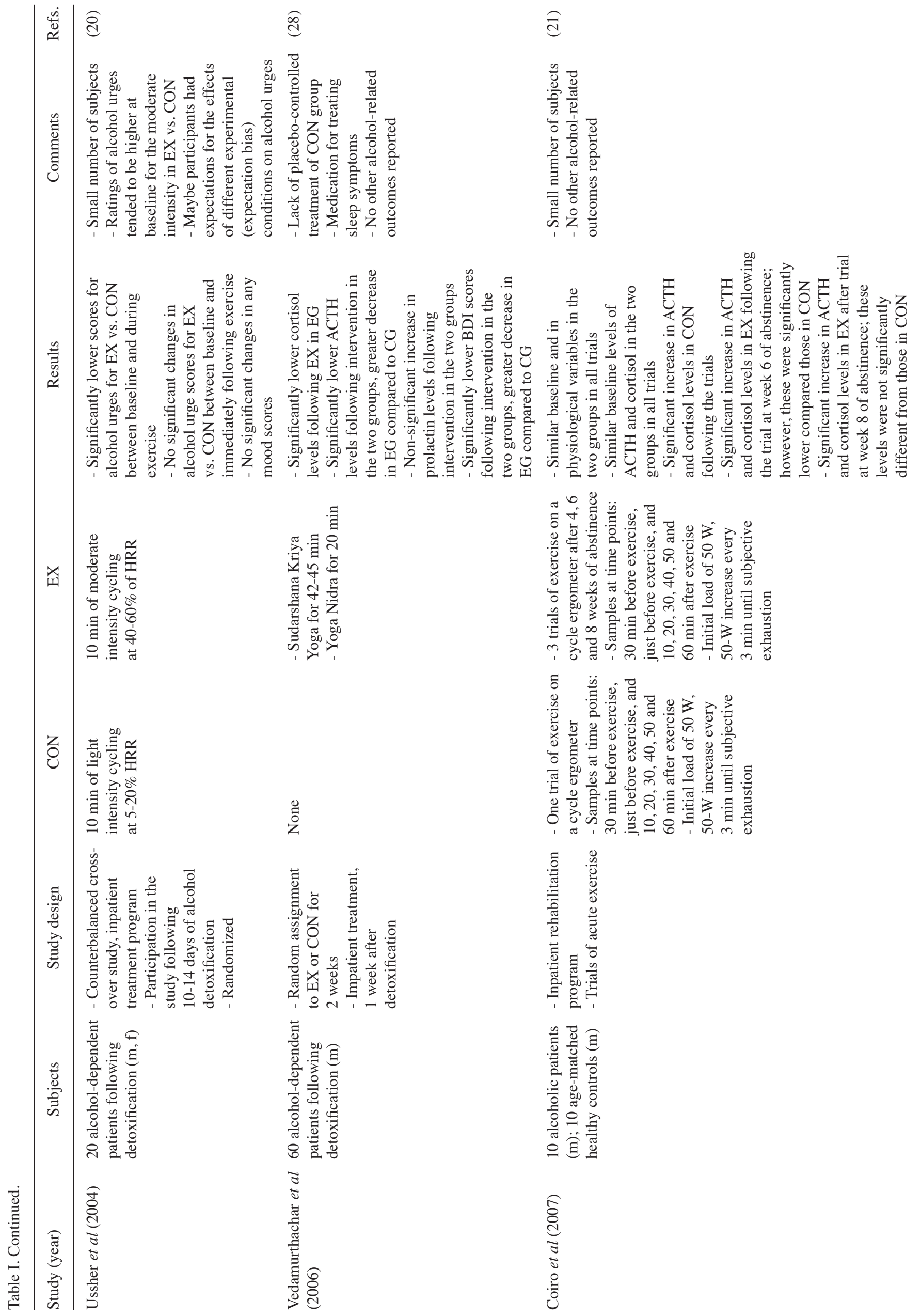




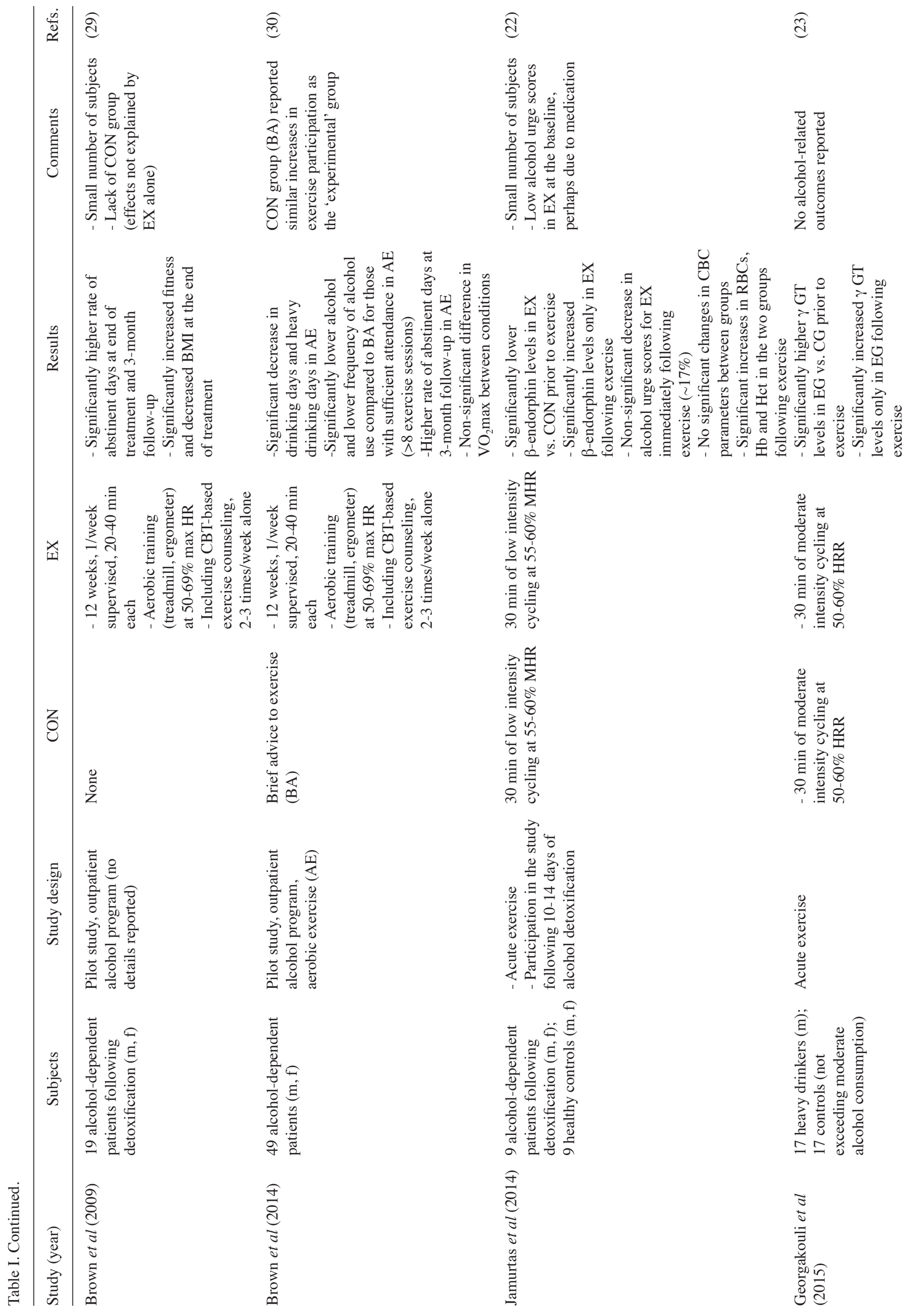


during exercise, but not following exercise. No difference in mood between the groups was observed throughout the study. It is not known, however, whether a higher intensity of exercise as well as longer duration would be able to induce further favourable effects on alcohol urges.

Coiro et al (21) investigated physiological and biochemical responses to acute exercise in 10 alcoholic patients after 4 , 6 and 8 weeks of alcohol abstinence. All the trials were conducted on a cycle ergometer, with an initial load of $50 \mathrm{~W}$ and a $50-\mathrm{W}$ increase every 3 min until subjective exhaustion. There was also a control group that performed only one trial. The results showed that baseline and exercise-induced changes in physiological variables were similar in the two groups in all trials. Concerning the biochemical variables, baseline levels of adrenocorticotropic hormone (ACTH) and cortisol in alcoholic patients were similar to those in the control group in all trials. Exercise resulted in significantly increased ACTH and cortisol levels in alcoholic patients after the trial at week 8 of abstinence, but not at the previous trials, and reached those observed in the control group. Despite the small number of subjects, the present study indicated that alcoholic patients have lower responses of ACTH and cortisol to acute exercise compared to healthy controls following detoxification; however, 8 weeks of abstinence from alcohol eventually leads these responses back to normal.

A more recent study by Jamurtas et al (22) used an exercise protocol of longer duration (30 $\mathrm{min})$ and low intensity ( $60 \%$ of the maximum heart rate). The results indicated an $18.6 \%$ decrease in alcohol urge in alcoholic patients compared to the control group that underwent the same exercise protocol. Although this change was not statistically significant, it was close to the difference noticed in the aforementioned acute experiment (20). Limitations of this study, such as the low number of participants, may be a reason for the lack of statistical significance. Regardless, to the best of our knowledge, this is the first study to explore the biochemical parameters associated with alcohol urge. $\beta$-endorphin levels were significantly lower in alcoholic patients at baseline, whereas $30 \mathrm{~min}$ cycling exercise resulted in a 3 -fold significant increase in the same group.

Another study by Georgakouli et al (23) examined the effect of acute exercise on indices of liver function and blood redox status in heavy drinkers and individuals that do not exceed the limits for moderate alcohol use (control group). The exercise protocol used in this study was a single trial of moderate intensity exercise (50-60\% of the heart rate reserve) for $30 \mathrm{~min}$ at an ergometer. The results showed that heavy drinkers may be prone to oxidative stress, as indicated by significantly decreased baseline reduced glutathione levels and also a trend for decreased baseline thiobarbituric acid reactive substances levels in heavy drinkers compared to controls. Exercise induced antioxidant responses were lower in heavy drinkers compared to controls. Concerning liver function, baseline $\gamma$ glutamyl transferase levels were significantly increased in heavy drinkers compared to controls. Furthermore, exercise led to increased aspartate aminotransferase levels in the two groups and increased alanine aminotransferase levels only in heavy drinkers. These results indicate that responses of liver enzymes to exercise may be influenced by the level of alcohol consumption. This study reports for the first time that heavy 
drinkers may respond differently to acute exercise compared to the individuals that do not drink heavily, highlighting that there is a requirement for more studies on the effect of exercise on indices of health status in this population.

Exercise training. Medium- and long-term intervention studies provide rather limited and contradictory results. In 1972, Gary and Guthrie (24) reported that self-esteem of alcoholic patients improved along with fitness following a 4-week jogging program, but there was no effect regarding drinking episodes. In a study by Sinyor et al (25), 58 individuals who were receiving treatment for alcohol dependence participated in a 6-week fitness program. Results showed that participants had significantly higher abstinence rates and fitness gains at 3 and 18 months following discharge when compared with a control group. However, this study had a number of methodological limitations. The most important was that the control group was formed by patients from different therapy centers receiving various treatments.

A study conducted by Murphy et al (26) assessed the effects of exercise and meditation on alcohol consumption in 48 college students who were heavy social drinkers. Participants were randomly assigned to three conditions: A no-treatment control group, an experimental group that participated in an 8 -week exercise program and a third group that participated in meditation training. Volunteers self-monitored their daily training program and consumption of alcohol throughout the study. The results showed that participants in the two treatment groups significantly reduced alcohol consumption by 14 drinks/week compared to the no-treatment control group by the end of the intervention. There were no dropouts during the exercise intervention, however, only $72 \%$ returned to the follow-up assessment at 6 weeks. It was also reported that participants in the experimental group increased their fitness level during intervention and also for 6 weeks following cessation of the intervention.

In 1997, Donaghy (27) investigated the effects of a 3-week supervised exercise program followed by a 12 -week home-based exercise program in 165 adults in an alcohol abstinence treatment program. Participants were randomly assigned into two groups; an experimental group, who undertook muscle stretching and aerobic exercise (AE), and a control group who undertook gentle stretching and breathing exercises 3 times a week. The results showed that participants in the experimental group improved fitness, strength, physical activity and physical self-perceptions during the exercise program, and they maintained these improvements for a further month by undertaking a home-based exercise program. However, only fitness was maintained after 5 months of follow-up, while the number of dropouts was high (26\% at month 1,30\% at month 2 and a further $17 \%$ at month 5). In addition, the relapse rates did not significantly differ between the groups throughout the study, indicating that this type of intervention may not induce significant changes in abstinence rates, depression and anxiety, despite the improvement in fitness.

Vedamurthachar et al (28) investigated the antidepressant effects of Sudarshana Kriya Yoga on alcohol-dependent inpatients. A total of 60 alcohol-dependent patients that had completed a detoxification program were separated into two groups; one group followed a 2-week program of Sudarshana
Kriya Yoga with a duration of 42-45 min (experimental group), whereas the other group received no treatment (control group). The results showed that intervention led to significantly decreased cortisol levels in the experimental group compared to the control group. However, decreased ACTH levels and Beck Depression Inventory scores were observed following intervention in the two groups. Certain limitations of this study were the lack of placebo-controlled treatment of the control group, medication for treating sleep symptoms in certain participants, and the lack of other alcohol-related outcomes.

More recently, Brown et al (29) developed a 12-week moderate intensity AE program as an adjunctive intervention for 19 alcohol-dependent patients in recovery. All the participants were receiving ongoing addiction treatment for alcohol dependence and they reported that their last drink was 0-58 days before the intervention. The results from this study showed a significantly higher rate of abstinence days at the end of the treatment and during a 3-month follow-up period, while no difference was found after the 3-month follow-up period. Furthermore, there was a significant increase in fitness and a decrease in body mass index at the end of treatment; however, no difference was found after the 3-month follow-up period. However, one of the drawbacks of this study was the lack of a control group.

In a follow-up study by the same investigators (30), 49 alcohol-dependent patients were separated to participate either in an AE program or in a brief advice (BA) to exercise condition. Participants in the AE group exercised once per week, for 12 weeks at an intensity corresponding to 50-69 of the maximal heart rate, while they also received exercise counseling 2-3 times per week. Participation in the exercise program resulted in a significant decrease in drinking days and heavy drinking days. Furthermore, participants with sufficient exercise attendance ( $>8$ exercise sessions) reported significantly lower alcohol consumption and lower frequency of alcohol use compared to BA. A higher rate of abstinent days at the 3-month follow-up assessment was reported for the AE group. However, it was reported that adherent AE participants had significantly greater heavy drinking days compared to BA participants during the follow-up period.

Based on the current limited literature, the efficacy of exercise in decreasing alcohol consumption cannot be established. Notably, the majority of the studies mentioned a positive attitude of participants towards drinking when they were systematically involved in exercise treatments. Although one study mentioned the involvement of the endogenous opioid system in the regulation of alcohol desire, mechanisms as well as the best type and mode of exercise underlying these effects remain to be elucidated.

Possible mechanisms of exercise effects on alcohol abstinence Psychological and physiological effects of exercise. Mechanisms that may render exercise useful in the treatment of AUDs are mainly psychological and physiological. Physical exercise is an effective mean of maintaining or enhancing overall health and wellness. It has been suggested that exercise has a dual beneficial role in the treatment of substance abuse. Firstly, it has the potential to attenuate the negative effects of ethanol consumption by mechanistic changes in the cell level. For example exercise may attenuate the ethanol-induced 
decline in hepatic mitochondria, extend oxidative damage in the body and may even ameliorate decreased capillarization in skeletal muscle (31). In regards to mental health, exercise has numerous beneficial effects on mood, anxiety, depression, self-perception and self-efficacy $(15,32,33)$. Additionally, exercise has been used as an adjunctive strategy for substance dependence during and following a treatment program (15). As was mentioned previously, exercise could be an effective treatment for AUDs; however, more research is required to elucidate the possible psychological and physiological mechanisms involved in the reduction of harmful alcohol consumption.

Psychological mechanisms. In the psychological domain, the theory of self-efficacy of Bandura (34) refers to the socio-cognitive mechanisms through which the individuals can regulate their behavior. The theory argues that the inner belief of an individual that makes them successfully carry out a particular behavior is associated with the ability to exhibit this behavior. As such, when people engage in exercise programs they increase their self-confidence and this process is further reflected to their everyday lives by enhanced levels of self-esteem and self-efficacy $(35,36)$. By analogy, this process may be transferred to the implementation of coping strategies necessary to stay away from alcohol.

A psychosocial mechanism that may be involved in alcohol abuse is the use of alcohol to deal with stress. Numerous people drink alcohol as they cannot cope with the stress of everyday life. However, exercise can reduce stress and thus may be used to reduce the desire for alcohol (37). Another important psychosocial mechanism proposed as a means to reduce alcohol abuse is that of social interaction. Based on this theory, social interaction during physical exercise may create strong associations among people who participate in it. Sometimes these associations may have a positive impact on mental health (38). It is suggested that physical activity can help improve the social support network as it can take the form of group activity and create a calm and conducive environment to rehabilitation. Additionally, it is an acceptable form of social support for addicted persons to substances under rehabilitation (39).

Depression is common among alcoholics (40). The beneficial effects of physical activity are well-documented in improving mood (41), fighting depression (42) and anxiety (43). Mobily et al (44) examined the effect of walking as a means of exercise on depression in people $>65$ years with moderate and severe depression. The results showed a positive impact of walking in people with serious disabilities and people with moderate depression and an even larger effect in people with severe depression. In their research Faulkner and Biddle (41) examined 6 cases of people suffering from clinical depression. Subsequent to applying a walking program, lasting $\geq 10$ weeks, the study reported that these people reduced their depressive mood and significantly improved their quality of life. Therefore, if physical activity can act positively on depression and anxiety it may function as a strategy for dealing with alcoholism as well.

In conclusion, taking into account the aforementioned mechanisms, the development of a healthy and physically active lifestyle may lead to improved health, quality of life and a form of positive behavior that one can adopt instead of alcohol abuse.

Physiological mechanisms. Exercise is associated with a euphoric feeling during and subsuequent to an exercise session $(45,46)$. It has been suggested that an improvement in the mood of heavy drinkers or alcoholics by an exercise-induced increase in $\beta$-endorphin levels, may act as an inhibitory factor to alcohol use. Therefore, a possible mechanism to explain the positive effects that exercise may have on alcohol abstinence and alcohol cravings is associated with the effects that exercise has on the endogenous opioid system. The functions of the endogenous opioid system mainly involve modulation of the response to pain, reward and reinforcement and regulation of functions such as thermoregulation and energy substrate mobilization (47-50). Pro-opiomelanocortin (POMC), as one of the three precursors of the endogenous opioid peptides, gives rise to $\beta$-endorphin, the main representative of the endogenous opioid system (49). Previous studies have indicated that physical activity affects the level of $\beta$-endorphin and its increase during exercise (51) is influenced by the intensity and duration of exercise $(45,46,52)$. $\beta$-endorphin is associated with the euphoria felt by the trainee during exercise. Furthermore, due to the fact that $\beta$-endorphin receptors are present in numerous areas of the body that participate in the metabolism of substances, $\beta$-endorphin is involved in the metabolism of carbohydrates during rest $(47,53)$ and exercise conditions (53-56).

The endogenous opioids are considered to be participating in the processes of tolerance to alcohol and abstinence from it. In particular, it is known that alcohol consumption increases the levels of $\beta$-endorphin that are associated with euphoria (57). Furthermore, it has been reported that the levels of $\beta$-endorphin are low in the initial phase of abstinence from alcohol (58). Analysis of $\beta$-endorphin and ACTH in cerebrospinal fluid in alcoholics showed that there is a lack of $\beta$-endorphin centrally with significantly increased levels of ACTH (59). These findings suggest that alcohol addiction may be associated with significant changes in POMC-related peptides. Furthermore, children of alcoholics had lower levels of $\beta$-endorphin compared to normal individuals, which were even lower when both parents were alcoholics (60), indicating that genetic factors may be involved in the phenomenon of alcoholism. The quantity and frequency of alcohol consumption is thought to induce different effects on the endogenous opioid system. Acute or light alcohol consumption results in greater $\beta$-endorphin levels, while chronic heavy alcohol consumption induces several neuronal systems to undergo adaptive changes in order to maintain their functional activity at normal levels, resulting in central opioid deficiency in the absence of alcohol (57). Thus, abstinence from alcohol in alcohol abusers/alcoholics has been associated with decreased levels of $\beta$-endorphin, which may result in alcohol withdrawal symptoms and may further promote alcohol consumption $(57,58)$.

In 2011, Zourbanos et al (61) proposed a model of the possible association between the desire for alcohol, $\beta$-endorphin, physical activity and the subsequent reduced desire for alcohol. This model is based on the rationale that alcohol and physical exercise increase levels of $\beta$-endorphin 
at the hypothalamic and peripheral levels and, therefore, affect and are affected by the opioid system. More specifically, as mentioned, those suffering from alcoholism have a decreased secretory capacity of $\beta$-endorphin, which may contribute to an increased desire for alcohol (62). Considering that physical exercise also increases the levels of $\beta$-endorphin, it may be assumed that any suitable volume of physical exercise may potentially lead to elevated levels of $\beta$-endorphin, which may substitute alcohol consumption and/or reduce the desire for it.

Additionally, it is known that the mesolimbic dopamine pathway partially mediates the reinforcing effects of ethanol, through interactions of certain opioid peptides with opioid receptors that increase dopamine release in the nucleus accumbens (63). Although the neuroanatomical locus of the reinforcing effects of exercise remains to be elucidated, data suggest that exercise generally activates the same reward pathways that are activated by ethanol or other addictive substances. For instance, acute exercise bouts increase dopamine concentrations $(64,65)$, and chronic exercise programs lead to sustained increases in dopamine concentrations (66). As the reinforcing properties of alcohol are mediated by increasing dopamine levels in mesolimbic and mesocortical pathways, exercise may be the tool through which changes in these pathways lead to reduced susceptibility in alcohol use. An example is given by the phenomenon known as exercise addiction.

Furthermore, ethanol consumption stimulates several other neuroendocrine responses in the HPA axis. Corticotropin releasing hormone $(\mathrm{CRH})$ is increased when alcohol is consumed, which stimulates POMC and consequently gives rise to $\mathrm{ACTH}, \beta$-lipotropin and $\beta$-endorphin. ACTH stimulates the cells of the adrenal cortex in order to increase the release of cortisol, which inhibits the release of $\mathrm{CRH}$ and ACTH through a negative-feedback mechanism. The release of $\mathrm{CRH}$ is also inhibited by opioidergic and $\gamma$-aminobutyric acid neurons and stimulated by serotonergic and noradrenergic neurons (49). The effects on neurotransmitters, which result in relaxation and calmness, drive psychological dependence and the person starts to believe that alcohol is necessary to help with daily distress. Over time, the neurons adjust and the new disturbed levels of neurotransmitters become the new normal. These data indicate that the HPA axis of alcoholic patients suffers reduced secretory capacity during abstinence from alcohol (21). Acute exercise does not increase levels of ACTH and cortisol in them to the same degree as in normal individuals (21). At least 1 month of abstinence from alcohol is required for the hormone levels to start returning to the normal levels. After 8 weeks of abstinence from alcohol, alcoholic patients may present the same response to hormonal parameters during physical activity (15 min of acute exercise of increasing intensity) as those in the not addicted individuals (21). This evidence suggests that in order to explore the biochemical changes associated with the HPA axis in alcoholic individuals during physical activity, longer interventions may be necessary.

\section{Conclusions}

In conclusion, evidence from existing studies suggests that exercise is a useful adjunctive tool to combat AUDs. It is perceived that physical activity can have a dual action upon alcoholism. Firstly, it may attenuate the negative effects of alcoholism on health while it may also act centrally on the neurotransmitter systems involved in the mechanisms of addiction. However, there is a lack of information on optimal intensity (high vs. low), duration (long vs. short), frequency and type (cardiovascular vs. resistance exercise or combination of the two) of exercise that may influence the opioid system and is required to help alcoholics abstain from alcohol. Another question is how much exercise these persons can withstand given that in certain instances exercise can be undesirable or painful.

Studies on hormones, peptides and neurotransmitter systems associated with the mechanisms of reward, reinforcement, alcohol addiction and their connection to exercise are scarce. Therefore, the acute exercise studies as well as chronic exercise interventions are required to provide support for the hypothesis that exercise can act as an alternative to alcohol use due to its similar action on distinct components of the central nervous system involved in the addictive processes. In addition, limited information exists regarding the effects of exercise in combination with other treatments against alcoholism and mainly pharmacological treatments. Finally, due to the promising effects of exercise on alcohol consumption, attempts should be made to delineate its possible role in prevention of alcoholism in heavy drinkers.

\section{Acknowledgements}

The present study was co-financed by the European Union [European Social Fund (ESF)] and Greek national funds through the Operational Program 'Education and Lifelong Learning' of the National Strategic Reference Framework (NSRF)-Research Funding Program: THALES. Investing in knowledge society through the European Social Fund.

\section{References}

1. Rehm J, Room R, van den Brink W and Jacobi F: Alcohol use disorders in EU countries and Norway: An overview of the epidemiology. Eur Neuropsychopharmacol 15: 377-388, 2005.

2. World Health Organization (WHO): Causes of death 2008: Data sources and methods. Department of Health Statistics and Informatics. WHO, Geneva, 2011.

3. World Health Organization (WHO): Global status report on alcohol and health. WHO, Geneva, 2011.

4. American Psychiatric Association (APA): Diagnostic and Statistical Manual of Mental Disorders (DSM-V). 5th edition. APA, San Francisco, CA, 2013.

5. Institute of Medicine: Broadening the base treatment for alcoholism. Wiley, New York, NY, 1990.

6. De Sousa A and De Sousa A: A one-year pragmatic trial of naltrexone vs disulfiram in the treatment of alcohol dependence. Alcohol Alcohol 39: 528-531, 2004.

7. Sinclair JD: Evidence about the use of naltrexone and for different ways of using it in the treatment of alcoholism. Alcohol Alcohol 36: 2-10, 2001.

8. Miller WR and Rollnick S (eds): Motivational interviewing: Preparing people for change. 2nd edition. Guilford, New York, NY, 2002.

9. Ryan RM and Deci EL: Self-determination theory and the facilitation of intrinsic motivation, social development, and well-being. Am Psychol 55: 68-78, 2000.

10. Project MATCH Research Group: Matching Alcoholism Treatments to Client Heterogeneity: Project MATCH posttreatment drinking outcomes. J Stud Alcohol 58: 7-29, 1997.

11. Project MATCH Research Group: Project MATCH secondary a priori hypotheses. Addiction 92: 1671-1698, 1997. 
12. Garbutt JC: The state of pharmacotherapy for the treatment of alcohol dependence. J Subst Abuse Treat 36: S15-S25, 2009.

13. Donaghy ME and Mutrie N: Is exercise beneficial in the treatment and rehabilitation of the problem drinker? A critical review. Phys Ther Rev 4: 153-166, 1999.

14. Donaghy ME, Ralston G and Mutrie N: Exercise as a therapeutic adjunct for problem drinkers. J Sports Sci 9: 440, 1991.

15. Read JP and Brown RA: The role of physical exercise in alcoholism treatment and recovery. Prof Psychol Res Pr 34: 49-56, 2003.

16. Kosmidou EB, Ioannidis TD, Lyssa V, Zisi V and Theodorakis Y: Examining alcohol and exercise students through Planned Behavior theory using self-identity and past behavior. Hell J Phys Educ Sport Sci 29: 272-289, 2009

17. Craft LL and Perna FM: The benefits of exercise for the clinically depressed. Prim Care Companion J Clin Psychiatry 6: 104-111, 2004.

18. Ekkekakis P: Let them roam free? Physiological and psychological evidence for the potential of self-selected exercise intensity in public health. Sports Med 39: 857-888, 2009.

19. Ekkekakis P and Acevedo EO: Affective responses to acute exercise: Toward a psychobiological dose-response model In: Psychobiology of physical activity. Acevedo EO and Ekkekakis P (eds). Human Kinetics, Champaign, IL, pp91-109, 2006.

20. Ussher M, Sampuran AK, Doshi R, West R and Drummond DC: Acute effect of a brief bout of exercise on alcohol urges. Addiction 99: 1542-1547, 2004.

21. Coiro V, Casti A, Jotti GS, Rubino P, Manfredi G, Maffei ML, Melani A, Volta E and Chiodera P: Adrenocorticotropic hormone/cortisol response to physical exercise in abstinent alcoholic patients. Alcohol Clin Exp Res 31: 901-906, 2007.

22. Jamurtas AZ, Zourbanos N, Georgakouli K, Georgoulias P Manthou E, Fatouros IG, Goudas M, Koutedakis Y and Theodorakis Y: Beta Endorphin and Alcohol Urge Responses in Alcoholic Patients Following an Acute Bout of Exercise. J Addict Res Ther 5: 194, 2014.

23. Georgakouli K, Manthou E, Fatouros IG, Deli CK, Spandidos DA, Tsatsakis AM, Kouretas D, Koutedakis Y, Theodorakis Y and Jamurtas AZ: Effects of acute exercise on liver function and blood redox status in heavy drinkers. Exp Ther Med 10 2015-2022, 2015.

24. Gary V and Guthrie D: The effect of jogging on physical fitness and self-concept in hospitalized alcoholics. Q J Stud Alcohol 33: 1073-1078, 1972

25. Sinyor D, Brown T, Rostant L and Seraganian P: The role of a physical fitness program in the treatment of alcoholism. J Stud Alcohol 43: 380-386, 1982.

26. Murphy TJ, Pagano RR and Marlatt GA: Lifestyle modification with heavy alcohol drinkers: Effects of aerobic exercise and meditation. Addict Behav 11: 175-186, 1986.

27. Donaghy ME: The investigation of exercise as an adjunct to the treatment and rehabilitation of the problem drinker (unpublished PhD thesis). University of Glasgow, glathesis: 1997-3250, 1997.

28. Vedamurthachar A, Janakiramaiah N, Hegde JM, Shetty TK, Subbakrishna DK, Sureshbabu SV and Gangadhar BN: Antidepressant efficacy and hormonal effects of Sudarshana Kriya Yoga (SKY) in alcohol dependent individuals. J Affect Disord 94: 249-253, 2006.

29. Brown RA, Abrantes AM, Read JP, Marcus BH, Jakicic J, Strong DR, Oakley JR, Ramsey SE, Kahler CW, Stuart G, et al: Aerobic exercise for alcohol recovery: Rationale, program description, and preliminary findings. Behav Modif 33: 220-249, 2009.

30. Brown RA, Abrantes AM, Minami H, Read JP, Marcus BH, Jakicic JM, Strong DR, Dubreuil ME, Gordon AA, Ramsey SE, et al: A preliminary, randomized trial of aerobic exercise for alcohol dependence. J Subst Abuse Treat 47: 1-9, 2014.

31. El-Sayed MS, Ali N and El-Sayed Ali Z: Interaction between alcohol and exercise: Physiological and haematological implications. Sports Med 35: 257-269, 2005.

32. Hughes JR: Psychological effects of habitual aerobic exercise: A critical review. Prev Med 13: 66-78, 1984.

33. Ekkekakis P and Petruzzello SJ: Acute aerobic exercise and affect: Current status, problems and prospects regarding dose-response. Sports Med 28: 337-374, 1999.

34. Bandura A: Self-efficacy: Toward a unifying theory of behavioral change. Psychol Rev 84: 191-215, 1977.
35. Landers DM and Arent SM: Physical activity and mental health. In: Handbook of sport psychology. 2nd edition. Singer RN, Hausenblas CM and Janelle HA (eds). Wiley, New York, pp740-765, 2001

36. Paluska SA and Schwenk TL: Physical activity and mental health: Current concepts. Sports Med 29: 167-180, 2000.

37. Monti PM, Rohsenow DR, Colby SM and Abrams DB: Coping and social skills training. In: Handbook of alcoholism treatment approaches: Effective alternatives. 2nd edition. Hester RK and Miller WR (eds). Allyn and Bacon, Needham Heights, MA, pp2221-2241, 1995 .

38. North TC, McCullagh P and Tran ZV: Effect of exercise on depression. Exerc Sport Sci Rev 18: 379-415, 1990.

39. Longabaugh R, Wirtz PW, Zweben A and Stout RL: Network support for drinking, Alcoholics Anonymous and long-term matching effects. Addiction 93: 1313-1333, 1998

40. Sher L: Depression and alcoholism. QJM 97: 237-240, 2004.

41. Faulkner G and Biddle S: Exercise and mental health: It's just not psychology! J Sports Sci 19: 433-444, 2001

42. O'Neal HA, Dunn AL and Martinsen EW: Depression and exercise. Int J Sport Psychol 31: 110-135, 2000.

43. O'Connor PJ, Raglin JS and Martinsen EW: Physical activity, anxiety and anxiety disorders. Int J Sport Psychol 31: 136-155, 2000.

44. Mobily KE, Rubenstein MM, Lemke JH, O'Hara MW and Wallace RB: Walking and depression in a cohort of older adults: The lower 65 rural health study. J Aging Phys Act 4: 119-135, 1996.

45. Farrell PA, Kjaer M, Bach FW and Galbo H: Beta-endorphin and adrenocorticotropin response to supramaximal treadmill exercise in trained and untrained males. Acta Physiol Scand 130: 619-625, 1987.

46. Goldfarb AH, Hatfield BD, Armstrong D and Potts J: Plasma beta-endorphin concentration: Response to intensity and duration of exercise. Med Sci Sports Exerc 22: 241-244, 1990.

47. Fatouros IG, Goldfarb AH, Jamurtas AZ, Angelopoulos TJ and Gao J: Beta-endorphin infusion alters pancreatic hormone and glucose levels during exercise in rats. Eur J Appl Physiol Occup Physiol 76: 203-208, 1997

48. Goldfarb AH and Jamurtas AZ: Beta-endorphin response to exercise. An update. Sports Med 24: 8-16, 1997.

49. Gianoulakis C: Influence of the endogenous opioid system on high alcohol consumption and genetic predisposition to alcoholism. J Psychiatry Neurosci 26: 304-318, 2001

50. Jamurtas AZ, Tofas T, Fatouros I, Nikolaidis MG, Paschalis V, Yfanti C, Raptis S and Koutedakis Y: The effects of low and high glycemic index foods on exercise performance and beta-endorphin responses. J Int Soc Sports Nutr 8: 15, 2011.

51. Goldfarb AH, Hatfield BD, Sforzo GA and Flynn MG: Serum beta-endorphin levels during a graded exercise test to exhaustion. Med Sci Sports Exerc 19: 78-82, 1987

52. Goldfarb AH, Hatfield BD, Potts $\mathrm{J}$ and Armstrong D Beta-endorphin time course response to intensity of exercise: Effect of training status. Int J Sports Med 12: 264-268, 1991.

53. Fatouros J, Goldfarb AH and Jamurtas AZ: Low carbohydrate diet induces changes in central and peripheral beta-endorphins. Nutr Res 15: 1683-1694, 1995.

54. Jamurtas AZ, Goldfarb AH, Chung SC, Hegde S and Marino C Beta-endorphin infusion during exercise in rats: blood metabolic effects. Med Sci Sports Exerc 32: 1570-1575, 2000.

55. Jamurtas AZ, Goldfarb AH, Chung SC, Hegde S, Marino C and Fatouros IG: Beta-endorphin infusion during exercise in rats does not alter hepatic or muscle glycogen. J Sports Sci 19: 931-935, 2001.

56. Jamurtas AZ and Fatouros IG: The effect of exercise on $\beta$-endorphin levels in blood. Inq Phys Educ Sports 2: 93-102, 2004.

57. Gianoulakis C: Endogenous opioids and addiction to alcohol and other drugs of abuse. Curr Top Med Chem 4: 39-50, 2004.

58. Inder WJ, Livesey JH and Donald RA: Peripheral plasma levels of beta-endorphin in alcoholics and highly trained athletes and the relationship to a measure of central opioid tone. Horm Metab Res 30: 523-525, 1998.

59. Genazzani AR, Nappi G, Facchinetti F, Mazzella GL, Parrini D, Sinforiani E, Petraglia F and Savoldi F: Central deficiency of beta-endorphin in alcohol addicts. J Clin Endocrinol Metab 55: 583-586, 1982

60. Del Arbol JL, Rico Irles J, Contreras I, Aguirre JC, Raya J, Ruiz Requena ME and Miranda MT: Plasma concentrations of beta-endorphins in the children of alcoholic patients. An Med Interna 24: 273-277, 2007 (In Spanish). 
61. Zourbanos N, Jamurtas A, Staveri E, Hatzigeorgiadis A and Theodorakis Y: Physical exercise as strategy in alcohol abuse treatment. Hell J Psychol 8: 123-145, 2011.

62. Esel E, Sofuoglu S, Aslan SS, Kula M, Yabanoglu I and Turan MT: Plasma levels of beta-endorphin, adrenocorticotropic hormone and cortisol during early and late alcohol withdrawal. Alcohol Alcohol 36: 572-576, 2001.

63. Koob GF: Drugs of abuse: Anatomy, pharmacology and function of reward pathways. Trends Pharmacol Sci 13: 177-184, 1992.

64. Meeusen R, Smolders I, Sarre S, de Meirleir K, Keizer H, Serneels M, Ebinger G and Michotte Y: Endurance training effects on neurotransmitter release in rat striatum: An in vivo microdialysis study. Acta Physiol Scand 159: 335-341, 1997.
65. Petzinger GM, Walsh JP, Akopian G, Hogg E, Abernathy A, Arevalo P, Turnquist P, Vucković M,Fisher BE,Togasaki DM, et al: Effects of treadmill exercise on dopaminergic transmission in the 1-methyl-4-phenyl-1,2,3,6-tetrahydropyridine-lesioned mouse model of basal ganglia injury. J Neurosci 27: 5291-5300, 2007.

66. FisherBE,PetzingerGM,NixonK,HoggE,BremmerS,MeshulCK and Jakowec MW: Exercise-induced behavioral recovery and neuroplasticity in the 1-methyl-4-phenyl-1,2,3,6-tetrahydropyridine-lesioned mouse basal ganglia. J Neurosci Res 77 : 378-390, 2004 